\title{
Apports En Carbone Et Azote Dans Le Fleuve Niger À Tondibia (Niamey) : Résultats De Deux (2) Ans D’observations
}

\author{
Alhou B. \\ Département de Sciences de la Vie et de la Terre, \\ Ecole Normale Supérieure, Université Abdou Moumouni, Niger \\ Issiaka Boukari \\ Département de Chimie, Ecole Normale Supérieure, \\ Université Abdou Moumouni, Niger
}

Darchambeau $F$.

Unité de chimie océanographique, Université de Liège, Belgique

doi: 10.19044/esj.2016.v12n21p167 URL:http://dx.doi.org/10.19044/esj.2016.v12n21p167

\begin{abstract}
The Niger River is the third more important river in Africa and drains a surface of about 2,120,000 $\mathrm{km}^{2}$. It includes six hydrographic regions representing West African ecosystems. Despite the importance of this river at the regional and continental scale, little information has been collected on its biogeochemical characteristics and particularly on its role in the transportation and the transformation of matter (carbon, nitrogen and phosphorus). This study present the results of two years investigation, April 2011 to March 2013 in the middle Niger, upstream Niamey (Niger) city $\left[2.01^{\circ} \mathrm{E}, 13.57^{\circ} \mathrm{N}\right]$, according to a bi-weekly observation frequency. The variables measured are temperature, conductivity, dissolved oxygen, $\mathrm{pH}$, suspended matter, dissolved inorganic carbon $(\mathrm{C})$ and nitrogen $(\mathrm{N})$ and particulars organics $\mathrm{C}$ and $\mathrm{N}$ as well as isotopic composition of these elements. Daily discharges of the river come from hydrologic station measurement of the Niger authority Basin (NBA) at Niamey city. Hydrologic situation was strongly contrasted between the 2 years of survey. The mean discharge of the first year was only $673 \mathrm{~m}^{3} \mathrm{~s}^{-1}$ (one of the weakest discharge recorded on the river Niger at Niamey since 1940), while the mean discharge of the second year was $1,096 \mathrm{~m}^{3} \mathrm{~s}^{-1}$. Our results show that suspended matter, particular organic carbon, dissolved organic carbon and dissolved inorganic carbon are transported mainly during the local flood, induced by precipitations in July and August. The second flood (Guinean flood) which occurred, during November to January, is characterized by low temperatures and clean waters.
\end{abstract}


Keywords : Niger River, Niamey, biogeochemistry, dissolved inorganic carbon, dissolved organic carbon and nitrogen

\section{Résumé}

Le fleuve Niger est le troisième plus important fleuve d'Afrique et draine une superficie d'environ $2120000 \mathrm{~km}^{2}$. Il englobe six régions hydrographiques caractéristiques des écosystèmes de l'Afrique occidentale. Nonobstant l'importance de ce fleuve à l'échelle régionale et continentale, peu de données ont été récemment collectées sur la biogéochimie du fleuve et en particulier sur son rôle dans le transport et la transformation de la matière, dont le carbone et l'azote. Cette étude traite des résultats d'un suivi sur deux années, d'avril 2011 à mars 2013 dans le Niger moyen, en amont de la ville de Niamey (Niger) $\left[2.01^{\circ} \mathrm{E}, 13.57^{\circ} \mathrm{N}\right]$, selon une fréquence d'observation bihebdomadaire. Les variables mesurées sont la température, la conductivité, l'oxygène dissous, le $\mathrm{pH}$, la matière en suspension (MES), les différentes formes du carbone $(\mathrm{C})$ et d'azote $(\mathrm{N})$ inorganiques dissous, le $\mathrm{C}$ et l' $\mathrm{N}$ organiques particulaires, ainsi que la composition isotopique de ces éléments. Les débits journaliers du fleuve proviennent de la station de mesure hydrologique de l'autorité du Bassin du Niger (ABN) à Niamey. La situation hydrologique a été fort contrastée entre les 2 années d'étude puisque le débit moyen sur la première année était de seulement $673 \mathrm{~m}^{3} \cdot \mathrm{s}^{-1}$, soit un des débits les plus faibles enregistrés sur le fleuve à Niamey depuis 1940 , et de $1096 \mathrm{~m}^{3} . \mathrm{s}^{-1}$, soit une valeur médiane, lors de la deuxième année. Ces résultats montrent que le carbone organique particulaire, le carbone organique dissous et le carbone inorganique dissous sont principalement transportés lors de la crue locale, engendrée par les précipitations de la mousson en juillet-août. La seconde crue, ou crue guinéenne, qui a lieu de novembre à janvier, est caractérisée par une chute des températures et des eaux claires.

Mots clés : Fleuve Niger, Niamey, biogéochimie, carbone et azote inorganiques dissous, carbone et azote organiques dissous

\section{Introduction}

Les écosystèmes aquatiques représentent, par rapport aux écosystèmes terrestres, de faibles superficies. Néanmoins, ils ne sont pas à négliger dans le bilan global en carbone qu'ils peuvent influencer au niveau régional (Cole et al., 2007). Ce qui n'est pas le cas dans les différents bilans établis bien que, le flux net en carbone de ces écosystèmes par unité de surface tend à être plus élevé comparativement aux apports provenant des terres environnantes. Plusieurs études n’ont pas manqué d'évaluer les flux en 
carbone au niveau régional des milieux aquatiques (Richey et al., 2002 ; Kortelainen et al., 2006 ; Algesten et al., 2004). Cependant, des efforts restent à faire pour mieux comprendre les relations entre les milieux aquatiques et terrestres en vue d'améliorer les estimations globales de carbone (Battin et al., 2008).

Mais, on retient que les écosystèmes aquatiques reçoivent du milieu terrestre du carbone sous forme organique ou inorganique avec beaucoup d'influence sur l'écologie et la dynamique du carbone dans le milieu récepteur (Caraco et Cole, 2004 ; Hanson et al., 2004 ; Garnier et al., 2013).

Malgré l'importance du fleuve Niger, les données relatives à ce dernier sur les différentes formes du carbone (C), et d'azote $(\mathrm{N})$ inorganiques dissous, le Cet l' $\mathrm{N}$ organiques particulaires, la composition isotopique de ces éléments sont quasi inexistantes ; ce qui constitue un vide scientifique. Quelques études par contre (Martins, 1983 in : Coynel et al., 2005 ; Picouet et al, 2002 ; Tardy et al, 2004) traitent des formes de carbone dans ce fleuve.

\section{Matériels et méthodes}

\section{Présentation de la zone d'étude}

Les échantillons d'eau sont prélevés sur le fleuve Niger en amont de la ville de Niamey à Tondibia (Figure 1) sous un climat aride caractérisé par une pluviométrie moyenne de $517,90 \mathrm{~mm}$, une température de $29,8^{\circ} \mathrm{C}$ et une évapotranspiration totale annuelle de $2802,5 \mathrm{~mm}$. Le site d'échantillonnage (Tondibia) se trouve à $185 \mathrm{~m}$ d'altitude entre 13³3'44"26 de latitude et $2^{\circ} 00$ '33"39 de longitude.

Le fleuve Niger, par sa longueur (4 $200 \mathrm{~km}$ ) représente le troisième grand fleuve d'Afrique. Il traverse le territoire du Niger sur près de $650 \mathrm{~km}$ et constitue le principal cours d'eau du pays. Son régime hydrologique est caractérisé par les hautes eaux causées par les quantités de pluies sur la partie haute de son bassin versant (Novembre à février), les basses eaux de mars à juin et une remontée faible des eaux à la suite des pluies locales et les affluents situés en rive droite de juillet à septembre. 


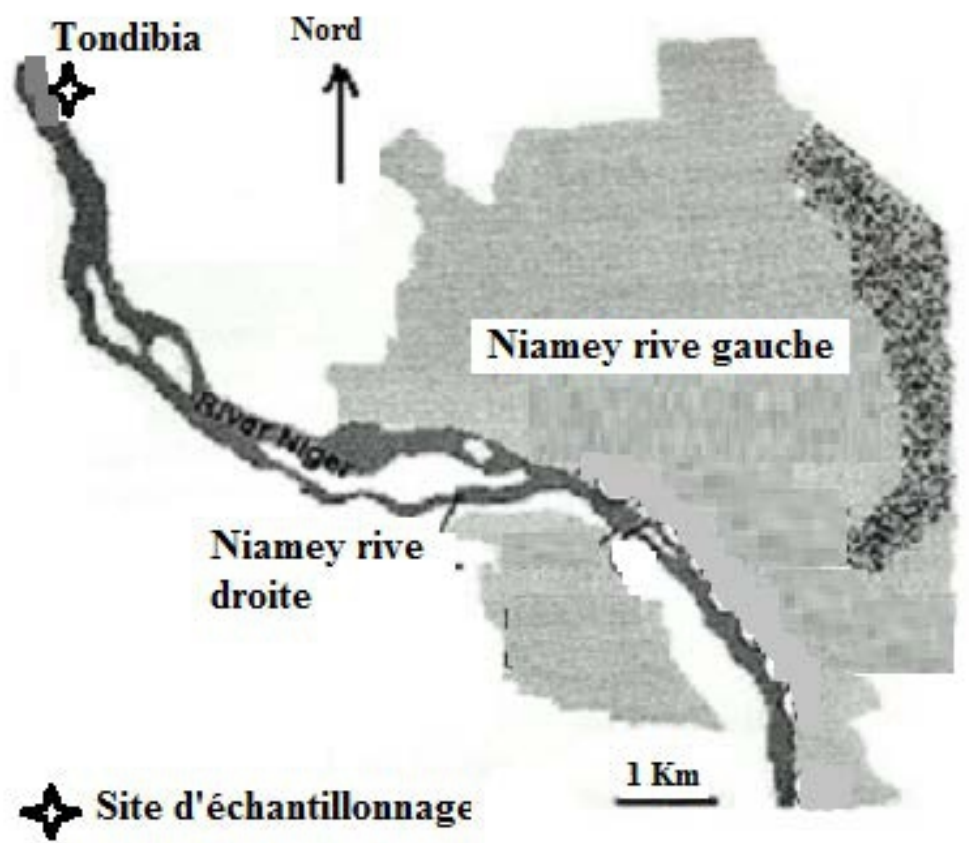

Figure 1 : localisation du site d'échantillonnage.

\section{Prélèvement, Conservation des échantillons et Techniques d'analyse}

Le suivi a été effectué sur le fleuve Niger en amont de la ville de Niamey d'avril 2011 à mars 2013 en raison d'un prélèvement d'eau toutes les deux semaines; soit un total de 50 échantillons prélevés. Ces prélèvements ont été effectués directement dans le fleuve à l'aide d'une bouteille de Van Dorn et mis dans des bouteilles soit en plastique ou en verre (après un triple rinçage). Ces bouteilles sont ensuite conservées au frais dans une glacière placée dans un véhicule jusqu'au retour au laboratoire.

La température, la conductivité, l'oxygène dissous et le $\mathrm{pH}$ sont mesurés in situ à l'aide d'une sonde multi-paramètres de marque HANNA.

Pour l'analyse des autres paramètres (les matières en suspension, les différentes formes de carbone et d'azote), les échantillons sont acheminés à l'université catholique de Louvain et à l'université de Liège.

Des échantillons d'eau pour l'analyse du $\square^{13} \mathrm{C}_{C I D}$ sont prélevés à partir de la bouteille de Van Dorn par un remplissage lent sans créer des bulles d'air de 2 Exetainers en verre de $12 \mathrm{ml}$, fixés avec $20 \square \mathrm{l}$ de solution saturée de $\mathrm{HgCl}_{2}$. Les Exetainers sont fermés avec leur bouchon blanc puis conservés à l'obscurité et à température ambiante.

Pour l'analyse du $\square{ }^{13} \mathrm{C}_{C I D}$, environ $300 \square \mathrm{L}$ de $\mathrm{H}_{3} \mathrm{PO}_{4}$ sont ajoutés pour convertir toutes sortes de carbone inorganique en $\mathrm{CO}_{2}$. Après toute une nuit de stabilisation, une partie est injectée dans un courant d'hélium d'un analyseur élémentaire, le spectrophotomètre de masse (EA-IRMS, 
ThermoFinnigan Flash 1112 et ThermoFinnigan Delta+XL) pour les mesures du $\square{ }^{13} \mathrm{C}$. Les données obtenues du $\square{ }^{13} \mathrm{C}$ sont corrigées grâce à l'équilibration isotopique entre les gaz et le $\mathrm{CO}_{2}$ dissout tel que décrit par Gillikin et Bouillon (2007).

Pour analyser les COD et $\delta^{13} \mathrm{C}_{\mathrm{COD}}$, un échantillon de $50 \mathrm{ml}$ de filtrat est obtenu à l'aide d'une seringue de $50 \mathrm{ml}$ surmontée d'un filtre encapsulé. L'échantillon a été conservé dans une bouteille en verre Supelco avec bouchon noir et septum, dans laquelle 50-100 $\square \mathrm{l}$ de $\mathrm{H}_{3} \mathrm{PO}_{4}$ ont été additionnés et le tout gardé à l'obscurité et à température ambiante. COD et $\delta^{13} \mathrm{C}_{\mathrm{COD}}$ ont été mesurées avec un analyseur de TOC OI-1010 couplé à un Thermo DeltaPlus IRMS par le même procédé que celui de St-Jean, 2003 et d'Osburn et St-Jean, 2007. La reproductibilité typique était <5\% pour COD, et $\pm 0,2 \%$ o pour $\delta^{13} \mathrm{C}_{\mathrm{COD}}$.

Des échantillons pour MES ont été prélevés A partir du contenu de la bouteille Van Dorn. Ils sont filtrés immédiatement sur le champ à l'aide des filtres Whatman GF/F $47 \mathrm{~mm}$, pré-pesés et préchauffés (toute la nuit à $450^{\circ} \mathrm{C}$ ), puis séchés par la suite.

A partir du contenu de la bouteille Van Dorn des échantillons ( 100 $\mathrm{ml}$ ) pour $\mathrm{COP}, \mathrm{NP}$ et $\delta^{13} \mathrm{C}_{\mathrm{COP}}$ ont été filtrés sur des filtres préchauffés Whatman GF/F $25 \mathrm{~mm}$ et séchées. Ces filtres ont été ensuite placés dans l'étuve à $60^{\circ} \mathrm{C}$ pour 48 heures minimum.

COP, NP et $\square^{13} \mathrm{C}_{\mathrm{COP}}$ ont été déterminés sur le EA-IRMS en utilisant le signal TCD du EA pour quantifier COP et NP, et en surveillant $\mathrm{m} / \mathrm{z} 44,45$ et 46 sur les IRMS.

De l'acétanilide a été utilisé comme norme pour COP et NP, tandis que le saccharose (AIEA-C6) a été utilisé pour calibrer les données de $\delta^{13} \mathrm{C}_{\text {COP. }}$

\section{Résultats}

\section{Paramètres physico-chimiques}

Le tableau 1 présente les résultats sur l'hydrologie du fleuve Niger et les caractéristiques physico-chimiques généraux de ses eaux.

Tableau 1 : Paramètres physico-chimiques et biologiques suivis d'avril 2011 à mars 2013 (n = nombre d'échantillons)

\begin{tabular}{|c||c|c||c||c|c|}
\hline $\mathrm{n}=50$ & $\begin{array}{c}\text { Débits } \\
\left(\mathrm{m}^{3} \mathrm{~s}^{-1}\right)\end{array}$ & $\begin{array}{c}\text { Température } \\
\left({ }^{\circ} \mathrm{C}\right)\end{array}$ & $\mathrm{pH}$ & $\begin{array}{c}\text { Oxygène Dissous } \\
(\% \text { de saturation })\end{array}$ & $\begin{array}{c}\text { Conductivité } \\
\text { Electrique } \\
\left(\mu \mathrm{S} \mathrm{cm}{ }^{-1}\right)\end{array}$ \\
\hline Moyenne & 885 & 26,55 & 7,49 & 91,92 & 53,42 \\
\hline \hline Écart type & 618 & 3,68 & 0,26 & 6,55 & 14,02 \\
\hline \hline Minimum & 75 & 18,51 & 7,05 & 70,90 & 35,00 \\
\hline \hline Maximum & 1963 & 31,10 & 8,01 & 103,50 & 102,00 \\
\hline
\end{tabular}




\section{Différentes formes de carbone}

- Carbone organique particulaire (COP)

Les concentrations en COP varient de 0,64 à $15,61 \mathrm{mg} \mathrm{L}^{-1}$. Ces concentrations augmentent de juin à septembre pour les deux années de suivi. Les plus faibles concentrations obtenues sont pratiquement similaires sur les deux années. Le taux le plus élevé en première année est de 10,22 mg L-1 obtenu en septembre ; il est de 15,61 $\mathrm{mg} \mathrm{L}^{-1}$ en juillet de la seconde année de mesure (Figure 2). Ce qui indique une variabilité annuelle des concentrations en COP.

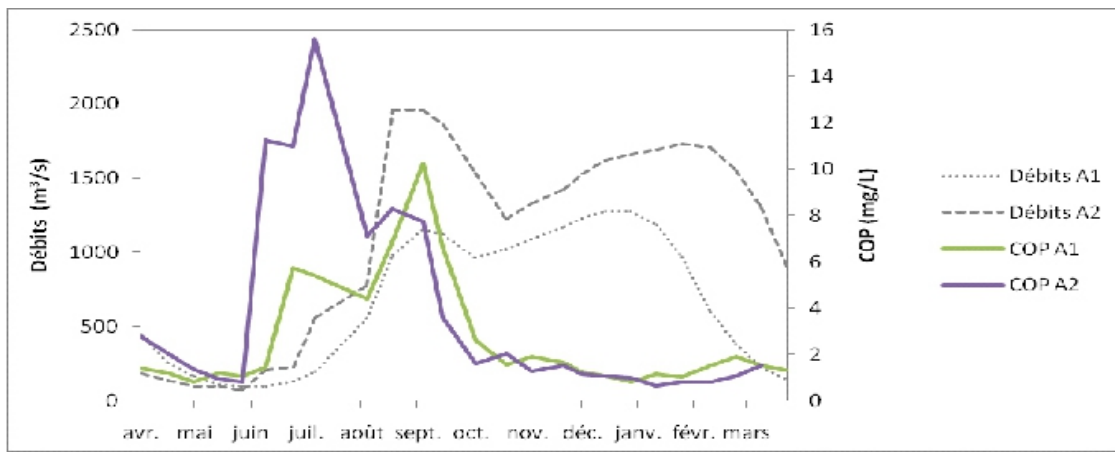

Figure 2 : Variation des concentrations en COP du fleuve Niger à Niamey d'avril 2011 à mars 2013.

- Carbone organique dissous (COD)

Les concentrations en COD du fleuve Niger varient entre 1,74 et 4,79 mg L-1 avec une moyenne de 2,74 mg L-1 sur les deux années de suivi. La valeur la plus faible est obtenue en août de l'année 1 et la plus élevée en septembre de l'année 2. Les pics de concentration apparaissent deux à trois mois avant la crue locale puis pendant cette crue avec un léger décalage en deuxième année. Il faut noter que les variations des concentrations sont assez faibles entre les deux années mais présentent néanmoins un effet saisonnier (Figures 3 et 4).

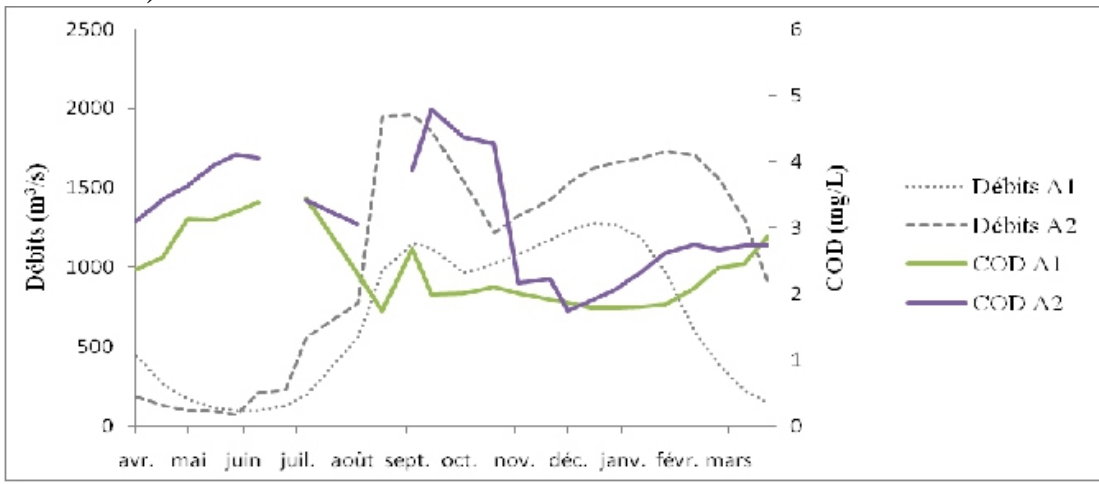

Figure 3 : Variation des concentrations en carbone organique dissous (COD) du fleuve Niger à Niamey d'avril 2011 à mars 2013 


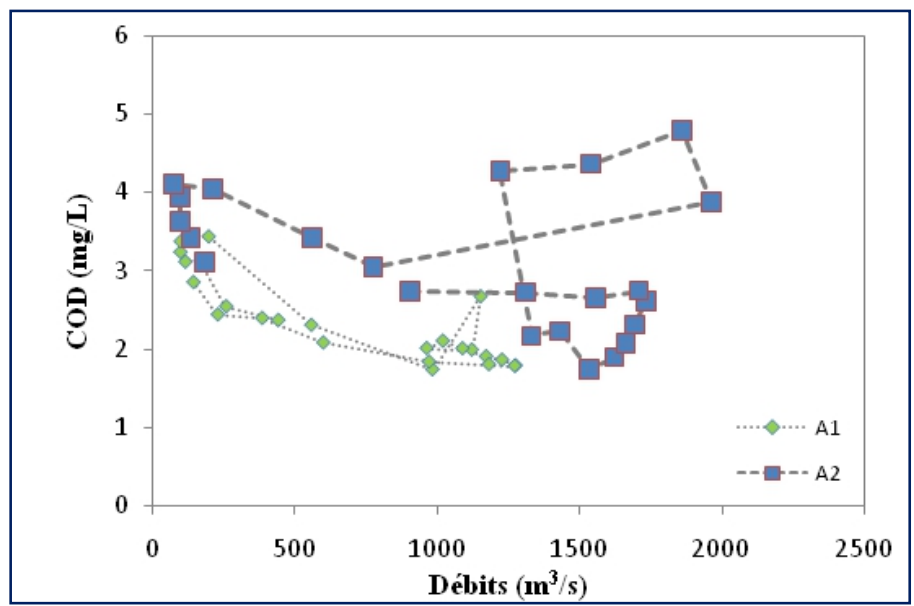

Figure 4 : interrelation entre COD et débits.

Le rapport COD/COP présente trois pic : un premier pic au mois de mai (3,9 et 5 mg L-1 en année 1 et 2 respectivement ; avant la saison des pluies), le second en octobre (1,3 et $2,7 \mathrm{mg}$ L- 1 en année 1 et 2 respectivement ; juste en fin de saison des pluies) et le troisième en janvier (2,1 et 3,6 $\mathrm{mg} \mathrm{L}-1$ en année 1 et 2 respectivement; pendant la crue guinéenne) (Figure 5).

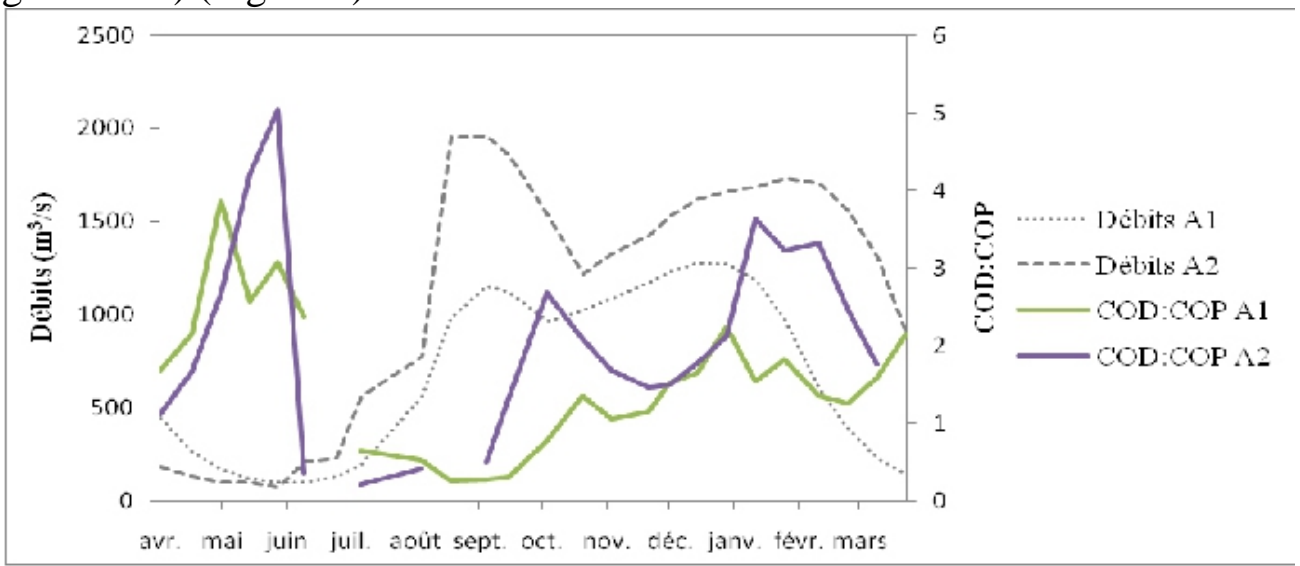

Figure 5 : Evolution du rapport COD/COP dans le fleuve Niger d’avril 2011 à mars 2013.

- Carbone inorganique dissous (CID)

Les concentrations en carbone inorganique dissous (CID) varient de $577 \mu \mathrm{M}$ (obtenu en octobre de la première année) à $9982 \mu \mathrm{M}$ (obtenu en mars de la deuxième année) avec une moyenne de $1731 \mu \mathrm{M}$ sur l'ensemble de la période d'étude (Figure 6). Ces concentrations ont significativement varié entre la première et la deuxième année passant d'une moyenne de 881 $\mu \mathrm{M}$ à $2472 \mu \mathrm{M}$, respectivement. 


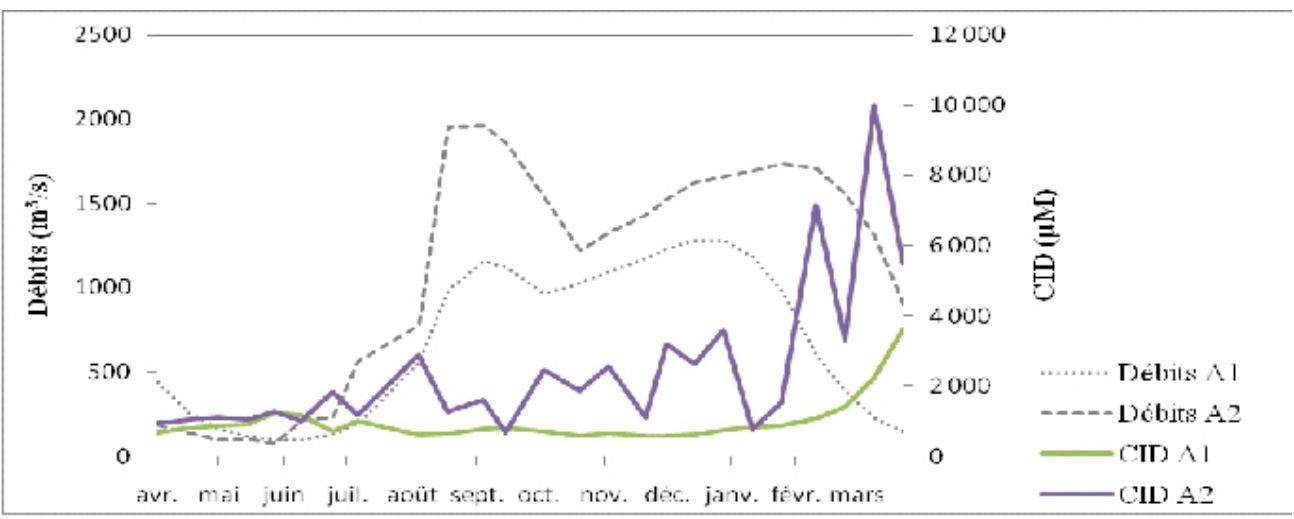

Figure 6 : Variation des concentrations en CID du fleuve Niger à Niamey d'avril 2011 à mars 2013.

Elles connaissent également une augmentation en fin de crue guinéenne et présentent des fluctuations beaucoup plus marquées en deuxième année sur l'ensemble de la période de suivi. Alors qu'en première année, la variation des concentrations en CID est relativement stable. La fluctuation des concentrations, en deuxième année, débute à la montée des eaux de la crue locale et persiste jusqu'à la fin de la crue guinéenne.

\section{- Azote particulaire (NP)}

La concentration en azote particulaire du fleuve Niger en amont de Niamey varie entre 0,07 et 2,20 mg L-1 avec une moyenne de 0,42 mg L-1 (Figure 7 ). L'isotope stable $\delta 15 \mathrm{~N}-\mathrm{NP}$ a une variation relativement faible $(0,07$ à $7,32 \%)$.

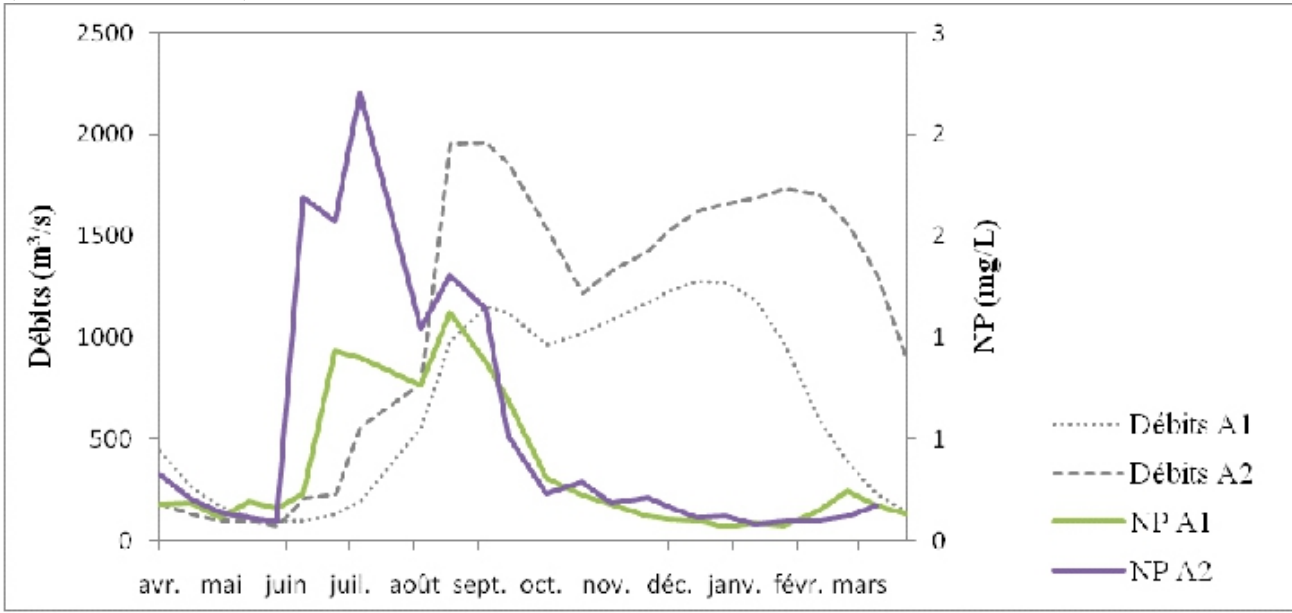

Figure 7 : Variation des concentrations en NP du fleuve Niger à Niamey d’avril 2011 à mars 2013.

- Matières en suspension (MES)

Les concentrations en MES varient de 18,2 à 1578,7 mg L-1 avec une 
moyenne de 223,8 $\mathrm{mg} \mathrm{L}^{-1}$. Les concentrations les plus élevées sont obtenues en saison des pluies (902,7 mg L-1 en août au cours de la première année et 1578,7 mg L-1 en juillet de la deuxième année) tandis que les plus faibles sont obtenues en étiage ( $21 \mathrm{mg} \mathrm{L-1}$ en mai pour la première année) et juste en fin de période de crue (18,2 mg L-1 en juin pour la deuxième année) (Figure 8).

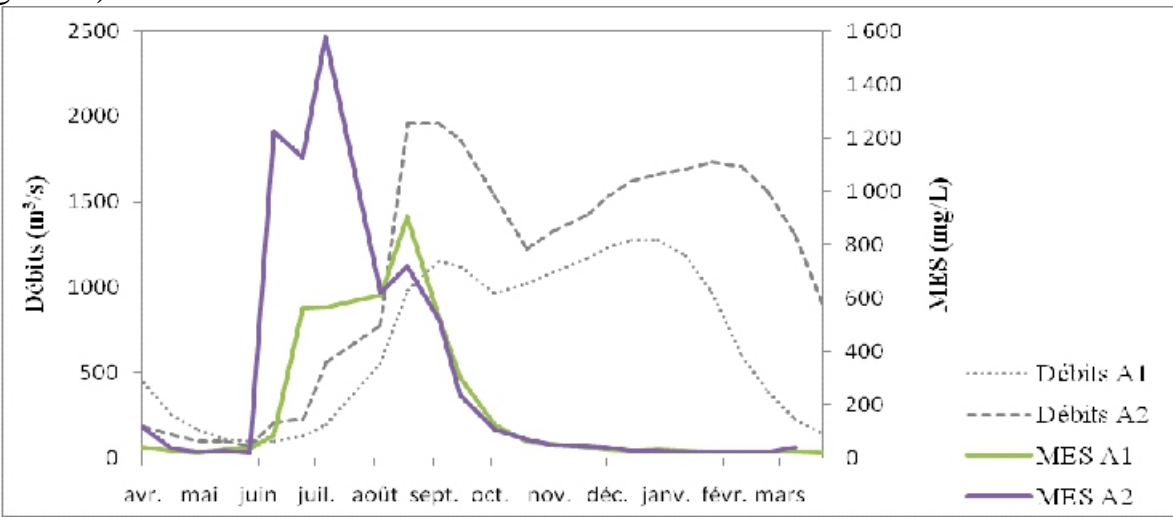

Figure 8 : Variation des concentrations en MES du fleuve Niger à Niamey d'avril 2011 à mars 2013

\section{- Azote inorganique dissous (NID)}

La concentration en azote inorganique dissout des eaux du fleuve Niger en amont de Niamey varie entre 0,2 et 111,6 $\mu \mathrm{M}$ avec une moyenne de 14,8 $\mu \mathrm{M}$ (Figure 8). Elle est plus élevée en début de la crue locale attestant ainsi les premiers apports provenant des eaux de ruissellement et plus faible en hautes eaux à cause du phénomène de dilution.

Le NID présente une dynamique similaire à celle des nitrates (Figure 9) ; ce qui signifie que les nitrates constituent la plus importante part d'azote inorganique dissous dans ce fleuve.

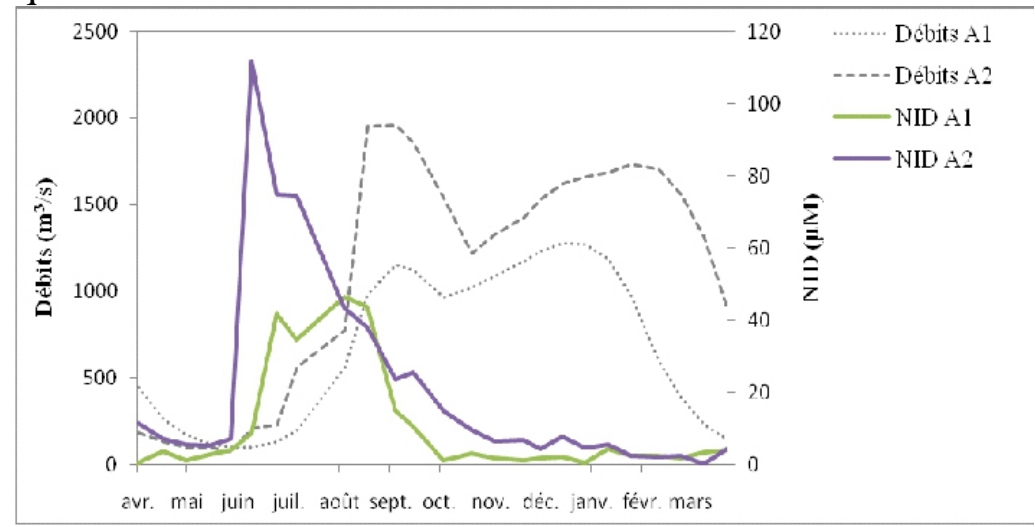

Figure 9 : Variation des concentrations en NID des eaux du fleuve Niger en amont de Niamey d'avril 2011 à mars 2013. 


\section{Discussions}

\section{Carbone Organique Particulaire (COP)}

La variabilité annuelle des concentrations en COP dans le fleuve Niger à Niamey n’est pas déterminée par le régime hydrologique du fleuve mais par les apports des eaux de pluies en saison hivernale qui va de juin à septembre. En effet, on note une synchronisation entre le début de la saison des pluies (crue locale) et l'augmentation des concentrations en COP. Pendant la crue guinéenne du fleuve Niger à Niamey, les concentrations sont relativement stables et varient entre 0,8 et $2 \mathrm{mg} . \mathrm{L}^{-1}$ sur les deux années. Cette tendance se confirme bien avec le $\delta^{13} \mathrm{C}$-COP qui présente également un effet saisonnier (Figure 10).

Les concentrations en COP en amont de Niamey sont plus élevées que celles du Niger supérieur $\left(0,64\right.$ à $15,61 \mathrm{mg} . \mathrm{L}^{-1}$ contre 0,1 à $1,85 \mathrm{mg} . \mathrm{L}^{-1}$; Picouet, 1999).

Il faut noter qu'en dehors de la saison des pluies où le Niger à des concentrations importantes, les concentrations dans les fleuves Niger, Sénégal (Gac et Kane, 1986) et Gambie (Lesack et al., 1984; Meybeck et al., 1987) sont semblables.

Par contre la comparaison avec l'Oubangui et le Mpoko, donne un taux de COP plus élevé pour l'Oubangui (11,5\%), et plus faible pour Mpoko (4,2\%) (Coynel et al, 2005).

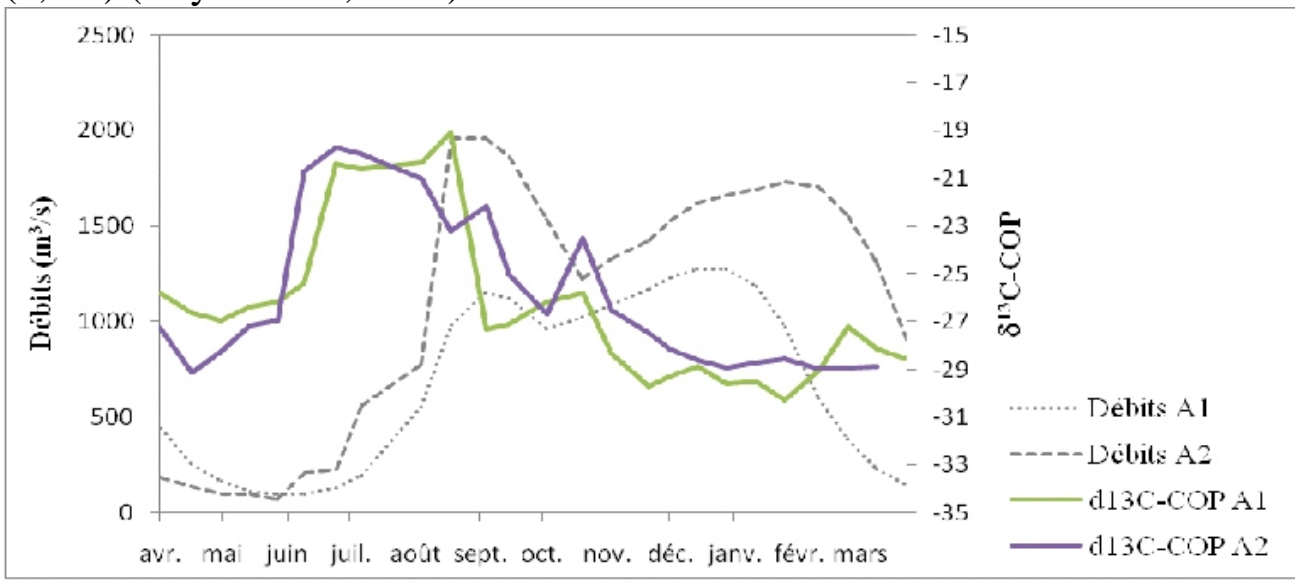

Figure 10 : Variation des taux en isotope $\delta^{13} \mathrm{C}$-COP du fleuve Niger à Niamey d'avril 2011 à mars 2013.

La figure 11 ci-dessous montre une interrelation négative très significative entre le \% COP et le MES $(\mathrm{R}=-0,652 ; \mathrm{P}<0,01)$. Ce type d'interrelation est rapporté par plusieurs auteurs (Meybeck, 1982, Picouet, 1999, Wasson et al., 1991, Meybeck et al., 1996b :In Picouet, 1999, Veyssy, 1998) dans d'autres rivières à travers le monde. 


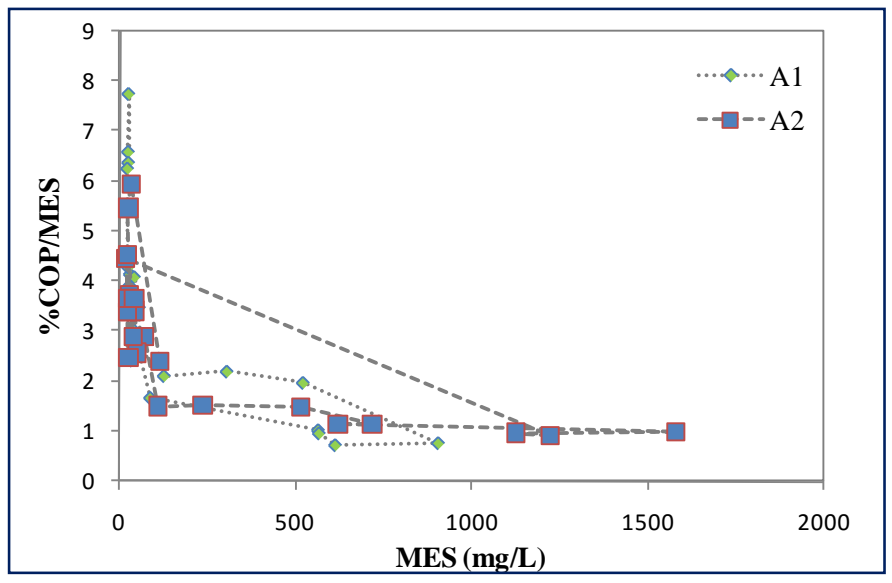

Figure 11 : Interrelation MES et \% COP

Ce résultat obtenu, dans un contexte où les apports des eaux de ruissellement provenant des sols dégradés sont importants pendant les pluies locales, s’explique par le mélange entre les apports des eaux de ruissellement des sols riches en matière organique et/ou des contributions directes de la litière, plus des sédiments provenant des sols profonds avec la charge organique en C réduite (Thurman, 1985 et Ludwig et al., 1996 cités par Tamooh et al., 2012).

En effet, la corrélation entre le \% COP et le rapport COP: NP $(\mathrm{R}=0,489 ; \mathrm{P}<0,01)$ confirme un apport allochtone de la majorité du carbone organique dans le fleuve Niger (Tamooh et al., 2012).

\section{Carbone Organique Dissous (COD)}

Plusieurs études sur d'autres cours d'eau (Boyer et al., 1996; Royer et David, 2005; Dalzell et al., 2007; Morel et al., 2009; Sanderman et al., 2009) rapportent une augmentation du COD en fonction du débit. On observe une toute autre situation sur le fleuve Niger où les concentrations en COD sont plus élevées, en mai et juillet, pendant que le débit du fleuve est très bas. Cette forte augmentation des concentrations en COD avant les deux crues du fleuve s’expliquerait par des pluies locales précoces (mai, juin) alors que la crue locale n’atteint son maximum qu'en août et la crue guinéenne en décembre.

Les concentrations en COD du fleuve Niger sont très proches des valeurs moyennes des rivières traversant les climats semi-arides comme rapporté par Spitzy et Leenheer (1991). La corrélation entre $\delta 13 C$-COD et $\delta 13 \mathrm{C}-\mathrm{COP}$ n'étant pas significative, cela suppose que l'échange de $\mathrm{C}$ entre les deux formes de carbone est très limité (Tamooh et al., 2012 ; Middelburg et Herman, 2007). Ainsi, la variation saisonnière et annuelle dans la signature isotopique du $\delta 13 \mathrm{C}$-COD qui indique vraisemblablement une origine variée 
du COD selon l'hydrologie du fleuve (Figure 14).

Le $\delta 13 C-C O D$ dans le fleuve Niger (Figure 13) présente une gamme de variation plus étendue que le fleuve Oubangui (-31 à -22\%o contre -32 à $27 \%$ ) ; cette large variation de la signature isotopique fait penser à des apports simultanés d'origine végétale de type C3 et C4 (Bouillon et al., 2012).

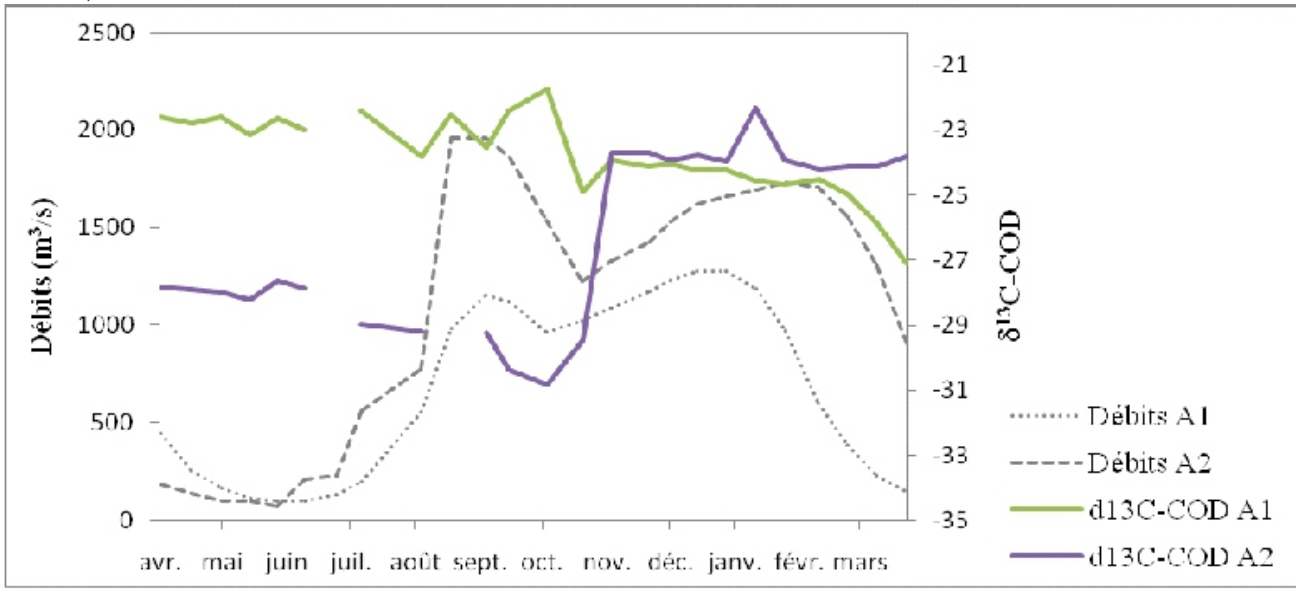

Figure 12 : Variation des taux en $\delta^{13} \mathrm{C}-\mathrm{COD}$ dans le fleuve Niger à Niamey d'avril 2011 à mars 2013

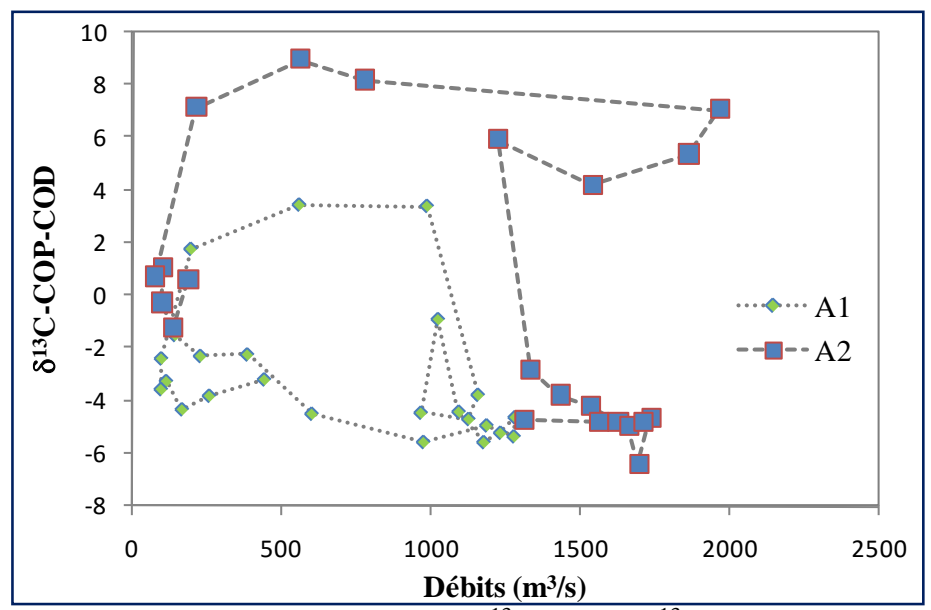

Figure 14 : interrelation entre Delta $\left(\delta^{13} \mathrm{C}-\mathrm{COP}-\delta^{13} \mathrm{C}-\mathrm{COD}\right)$ et les débits. 


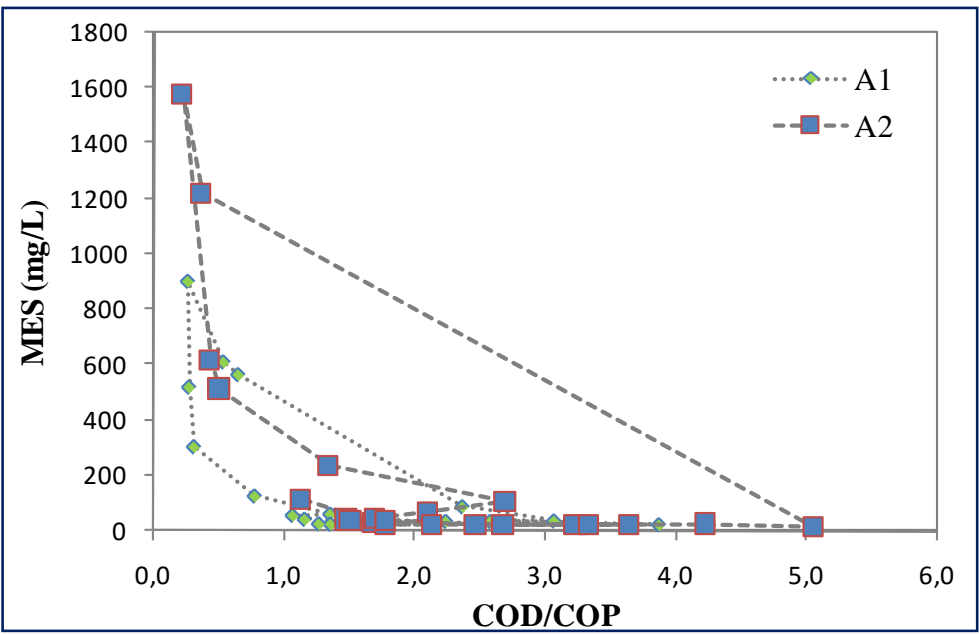

Figure 15 : interrelation entre MES et COD/COP

\section{Carbone Inorganique Dissous (CID)}

La variation interannuelle et intra-annuelle (particulièrement en deuxième année) des concentrations en CID provient probablement du caractère exceptionnel du régime hydrologique du fleuve qui déborde de son lit inondant ainsi les quartiers, les aménagements agricoles et les sites maraîchers. En effet, l'interrelation négative et très significative $(\mathrm{P}<0,01)$ entre le taux en $\delta 13 \mathrm{C}$ - CID et les concentrations en MES (Figure 16) témoigne de cette variabilité.

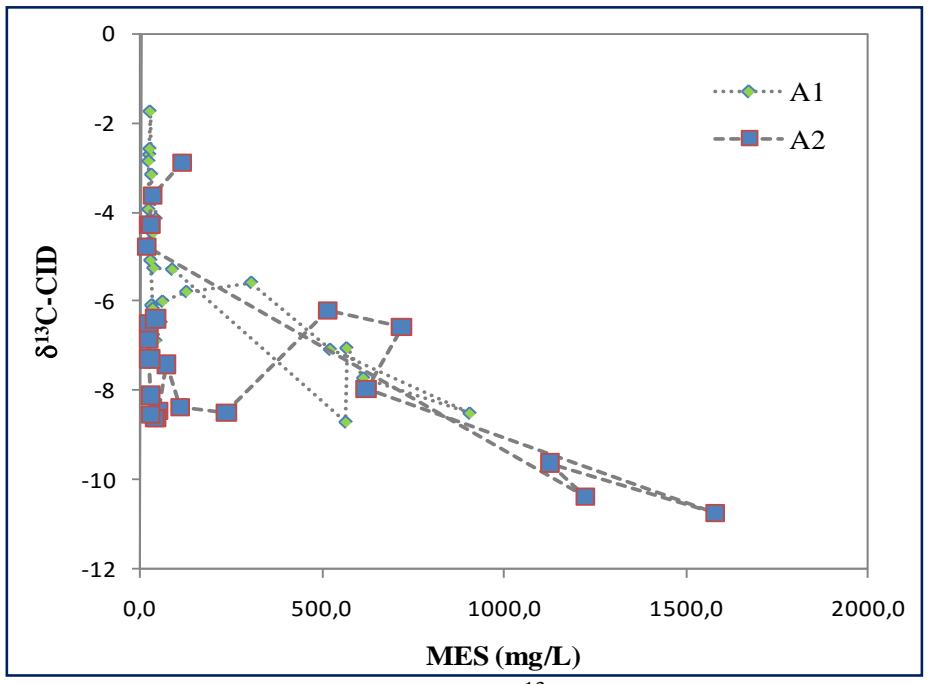

Figure 16 : Interrelation $\delta^{13} \mathrm{C}$ - CID et MES.

Les valeurs moins négatives par rapport aux gammes de variation de $\delta 13 \mathrm{C}$ pour le sol et les plantes en $\mathrm{C} 3$ et $\mathrm{C} 4$. Ce qui laisse penser à une contribution du CO2 atmosphérique (moyenne $\delta 13 \mathrm{C}$ atmosphérique $=-8 \%$; 
Brunet, 2004) dans la composition en CID de ce fleuve en plus des plantes en $\mathrm{C} 4$.

Par ailleurs, la corrélation très faible entre $\delta^{13} \mathrm{C}-\mathrm{CID}$ et $\mathrm{Ca} / \mathrm{Si}$ pourrait s'expliquer par la prédominance des fluctuations de $\mathrm{CO}_{2}$ sur les variations de $\delta^{13} \mathrm{C}$ - CID comme indiqué par Bouillon et al, 2012. Cette contribution en $\mathrm{CO}_{2}$ pourrait être plus importante en début de la crue locale qui est caractérisée par une diminution de signature isotopique du $\delta^{13} \mathrm{C}-\mathrm{CID}$, situation similaire à celle de l’Oubangui (Bouillon et al., 2012).

\section{Azote Particulaire (NP)}

Le rapport COP/NP est largement supérieur au \%COP/MES. Ce résultat indique des concentrations en MES et NP respectivement supérieures et inférieures aux concentrations en COP. Ces résultats sont contraires à ceux obtenus par Marwick et al. (2013) sur le fleuve Kenya où le développement du phytoplancton est important. Généralement, dans les fleuves tropicaux on note une masse importante de phytoplancton en saison sèche, période pendant laquelle la turbidité est faible et le séjour de l'eau plus long (di Persia and Neiff, 1986; Koné et al., 2009, Dudgeon, 1995, Townsend et al., 2011).

La variation relativement faible de l'isotope stable $\delta^{15} \mathrm{~N}$-NP traduit une absence de saisonnalité dans la variation des concentrations en NP du fleuve Niger à Tondibia.

\section{Matières En Suspension (MES)}

Les écoulements d'eau terrestre jouent un rôle très important dans le transport de la matière solide arrachée aux sols dénudés du bassin du fleuve Niger vers celui-ci et dans la remise en suspension des sédiments. Cela s'observe parfaitement à travers les travaux de Picouet (1999) et Picouet et al (2002).

Ainsi, la crue locale constitue une source importante de MES pour le fleuve ; cela se manifeste par des pics élevés en cette période (Figure 8 et 17). Selon les années, ces pics peuvent apparaître en juillet ou en août selon la précocité de l'installation de la saison des pluies. Cette dernière est reconnue par certains auteurs comme un moment où les rivières tropicales sont chargées en particules provenant du ruissellement des eaux sur des terres érodées (Townsend-Small et al., 2007 ; Hilton et al., 2008 ; Olivier et al., 2008 ; Coynel et al., 2005; Hung et Huang, 2005). 


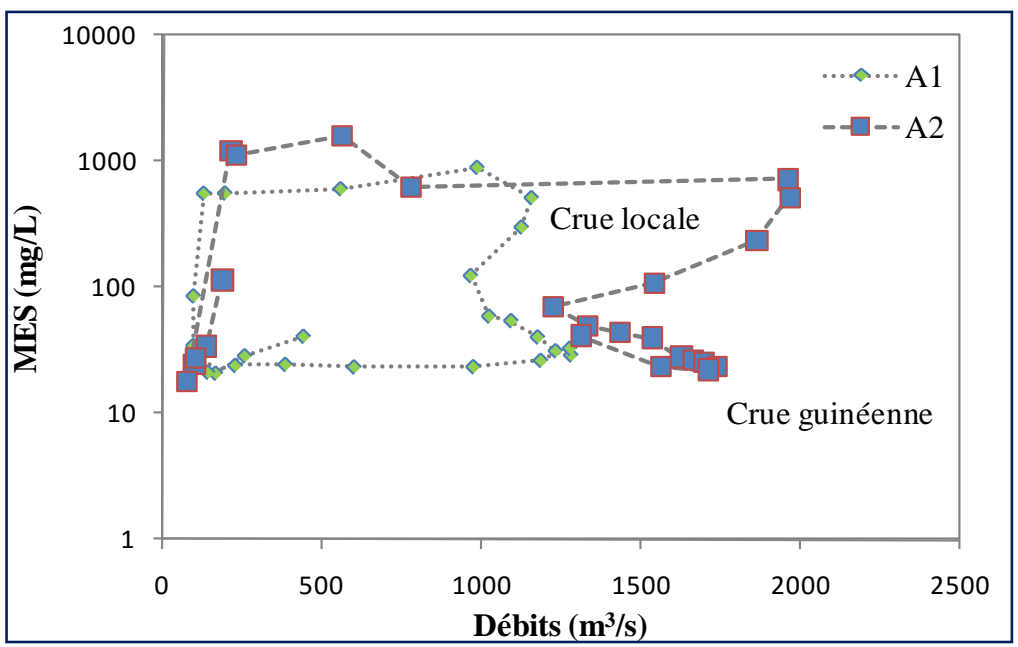

Figure 17 : interrelation entre MES et débits.

\section{Conclusion}

Les résultats de cette étude montrent clairement l'interrelation qui existe entre les écosystèmes terrestres et aquatiques. En effet, la dynamique biogéochimique des eaux du fleuve Niger à Tondibia (Niamey), notamment les fluctuations dans les concentrations des différentes formes de carbone et d'azote, est fortement influencée par les eaux de ruissellement qui drainent des vastes espaces dégradés dans le bassin du Niger. Ce dernier est essentiellement dominé par les plantes à C4 ; tandis que les plantes à C3 ont une présence saisonnière. Par sa particularité hydrologique (existence de deux crues), le fleuve Niger à Tondibia présente une dynamique contraire à celle de certains fleuves africains comme l'Oubangui. En effet, les concentrations en carbone et en azote ne suivent pas la variation du débit du fleuve ; elles sont surtout influencées par les premières pluies locales.

Remerciements. Au terme de cette étude, nous présentons nos remerciements :

- au projet AFRIVAL pour son appui financier dans la collecte des données et l'analyse des échantillons ;

- à l'autorité du bassin du Niger pour les données sur les débits du fleuve Niger à Niamey ;

- à la société d'exploitation des eaux du Niger pour les données sur le $\mathrm{pH}$ des eaux du fleuve.

\section{References:}

Algesten G., Wikner J., Sobek S., Tranvik LJ., Jansson M. 2004. Seasonal variation of $\mathrm{CO} 2$ saturation in the Gulf of Bothnia : Indications of marine net heterotrophy. Global Biogeochemical Cycles, 18(4), Art. no. GB4021. 
Battin TJ., Kaplan LA., Findlay S., Hopkinson CS., Marti E., Packman AI., Newbold JD., Sabater F. 2008. Biophysical controls on organic carbon fluxes in fluvial networks, Nature Geoscience, 1: 95-100.

Bouillon S., Yambélé A., Spencer R. G. M., Gillikin D. P., Hernes P. J., Six J., Merckx R., and Borges A. V., 2012. Organic matter sources, fluxes and greenhouse gas exchange in the Oubangui River (Congo River basin). Biogeosciences, 9, 2045-2062. doi:10.5194/bg-9-2045-2012

Boyer E. W., Hornberger G. M., Benkala K. E., Mcknigth D., 1996. Overview of a simple model describing variation of dissolved organic carbon in an upland catchment. Ecological Modeling 86, 183-188.

Brunet F., 2004. Variations de la signature isotopique $\delta 13 \mathrm{c}$ du carbone inorganique dissous dans les rivières et les fleuves. Thèse de doctorat unique. Université Toulouse III - Paul Sabatier UFR SVT, 270 p.

Brunet F., Dubois K., Veizer J., Ndondo G.R. N., Ngoupayou J.R. N., Boeglin J.L., Probst J.L., 2009. Terrestrial and fluvial carbon fluxes in a tropical watershed: Nyong basin, Cameroon. Chemical Geology 265, 563572.

Caraco N.F. et Cole J.J., 2004. When terrestrial organic matter is sent down the river: importance of allochthonous $\mathrm{C}$ inputs to the metabolism in lakes and rivers. In: Polis A, Power ME, Eds. Food webs at the landscape level. Chicago: University of Chicago Press. pp 301-16.

Caraco NF., Cole JJ. 2004. When terrestrial organic matter is sent down the river: importance of allochthonous $\mathrm{C}$ inputs to the metabolism in lakes and rivers. In: Polis A, Power ME, Eds. Food webs at the landscape level. Chicago: University of Chicago Press. pp 301-16.

Cole JJ., Prairie YT., Caraco NF., McDowell WH., Tranvik LJ., Striegl RG., Duarte CM., Kortelainen P., Downing JA., Middelburg JJ., Melack J. 2007. Plumbing the Global Carbon Cycle : Integrating Inland Waters into the Terrestrial Carbon Budget. Ecosystems 10:171-184.

Coynel A., Seyler P., Etcheber H., Meybeck M., et Orange D., 2005. Spatial and seasonal dynamics of total suspended sediment and organic carbon species in the Congo River. Global Biogeochemical Cycles, Vol. 19, GB4019, doi:10.1029/2004GB002335.

Dalzell, B.J., Filley T.R., Harbor J.M. 2007. The role of hydrology in annual organic carbon loads and terrestrial organic matter export from a Midwestern agricultural watershed. Geochimica et Cosmochimica Acta 71, 1448-1462.

di Persia D. H. and Neiff J. J., 1986. The Uruguay river system, in: The Ecology of River Systems, edited by: Davies, B. R., Walker, K. F., and Junk, W., Dordrecht, the Netherlands, 599-620.

Dudgeon D., 1995. The ecology of rivers and streams in tropical Asia, in: River and Stream Ecosystems, edited by: Cushing, C. E., Cummins K. W., and Minshall, G. W., Elsevier Science B.V., Amsterdam, 615-657. 
Gac J. Y., and Kane A.1986. Le fleuve Sénégal: I. Bilan hydrologique et flux continentaux de matières particulaires à l'embouchure, Science and Geology Bulletin, 39, 99 - 130.

Garnier J., Vilain G., Silvestre M., Billen G., Jehanno S., Poirier D., Martinez A., Decuq C., Cellier P., Abril G. 2013. Budget of methane emissions from soils, livestock and the river network at the regional scale of the Seine basin (France). Biogeochemistry, 1-16.

Gillikin D. P. and Bouillon S. 2007. Determination of $\delta 180$ of water and d13C of dissolved inorganic carbon using a simple modification of an elemental analyzer - isotope ratio mass spectrometer (EA-IRMS): an evaluation Rapid Communications in Mass Spectrometry, 21, 1475-1478.

Hanson PC., Pollard AI., Bade DL., Predick K., Carpenter SR., Foley JA. 2004. A model of carbon evasion and sedimentation in temperate lakes. Global Change Biology, 10:1285-98.

Hilton R. G., Galy A., Hovius N., Chen M. C., Horng M. J. and Chen H., 2008. Tropical-cyclone-driven erosion of the terrestrial biosphere from mountains, Nature Geoscience, 1, 759-762.

Hung J.J., Huang M.H. 2005. Seasonal variations of organic carbon and nutrient transport through a tropical estuary (Tsengwen) in southwestern Taiwan. Environmental Geochemistry and Health 27, 75-95.

Ittekkot, V. et Laane R. W. P. M. 1991. Fate of riverine particulate organic matter, in Biogeochemistry of Major World Rivers In: Coynel A., Seyler P., Etcheber H., Meybeck M., et Orange D., 2005, Spatial and seasonal dynamics of total suspended sediment and organic carbon species in the Congo River. Global Biogeochemical Cycles, Vol. 19, GB4019, doi:10.1029/2004GB002335.

Koné Y.J.M., Abril G., Kouadio K.N., Delille B., Borges A.V., 2009. Seasonal variability of carbon dioxide in the Rivers and Lagoons of Ivory Coast (West Africa). Estuaries and Coast, 32, 246-260. doi. 10-1007/s12237008-91-21-0

Kortelainen P., Rantakari M., Huttunen JT., Mattsson T., Alm J., Juutinen S., Larmola T., Silvola J., Martikainen PJ. 2006. Sedi-ment respiration and lake trophic state are important predic-tors of large $\mathrm{CO} 2$ evasion from small boreal lakes. Global Change Biology, 12:1554-67.

Lesack L. F. W., Hecky R. E. and Melack J. 1984. Transport of carbon, nitrogen, phosphorus and major solutes in Gambia River, West Africa, Limnology and Oceanography 29, $816-830$.

Ludwig W., Probst J. L. and Kempe S., 1996. Predicting the oceanic input of organic carbon by continental erosion, Global Biogeochem. Cy., 10, 23-41.

Martins, O., 1983. Transport of carbon in the Niger River, in Transport of Carbon and Minerals in Major World Rivers, vol. 55, edited by E. T. Degens, S. Kempe, and H. Soliman, pp. 435 - 449, Mitteilungen der Geologisch- 
Paläontologisches Institut, University of Hamburg, Germany.

Marwick T. R., Tamooh F., Ogwoka B., Teodoru C., Borges A. V., Darchambeau F. and Bouillon S. 2013. Dynamic seasonal nitrog en cycling in response to anthropog enic N-loading in a tropical catchment, AthiGalana-Sabaki River, Kenya. Biogeosciences Discussion, 10, 8637-8683. doi:10.5194/bgd-10-8637-2013.

MEYBECK M., 1982. Carbon, Nitrogen, and Phophorus transport by world rivers. American Journal of Science, Vol. 282, pp.401-450.

Meybeck M., Lô H. M., Cauwet G. and Gac J. Y. 1987. Geochemistry of the Sahelian Gambia River during the 1983 high-water stage, in Transport of Carbon and Minerals in Major World Rivers, vol. 64, edited by E. T. Degens, pp. 461 - 473, Mitteilungen der Geologisch-Paläontologisches Institut, University of Hamburg, Germany.

MEYBECK M., PASCO A., RAGU A., 1996. Evaluation des flux polluants dans les eaux superficielles. Etude inter-Agence de l'eau, Ministère de l'Environnement, 3 tomes.

Middelburg J. J. and Herman P. J. M., 2007. Organic matter processing in tidal estuaries, Marine Chemistry, 106, 127-147.

Morel B., Durand P., Jaffrezic A., Gruau G., Molenat J. 2009. Sources of dissolved organic carbon during stormflow in a headwater agricultural catchment. Hydrological Processes 23, 2888-2901.

Olivier H. R., Borges A. V., Dehairs F., Middelburg J.J., Bouillon S., 2008. Carbon biogeochemistry of the Betsiboka estuary (north-western Madagascar). Organic Geochemistry 39, 1649-1658.

Osburn C. L. and St-Jean G. 2007. The use of wet chemical oxidation with high-amplification isotope ratio mass spectrometry (WCOIRMS) to measure stable isotope values of dissolved organic carbon in seawater, Limnology and Oceanography-Methods, 5, 296-308.

Picouet C., Dupré B., Orange D., Valladon M., 2002. Major and trace element geochemistry in the upper Niger river (Mali): physical and chemical weathering rates and CO2 consumption. Chemical Geology 185: 93 - 124.

Picouet C.1999. Géodynamique d'un hydro-système tropical Peu anthropisé : Le Bassin supérieur du Niger et son delta intérieur. Thèse de doctorat. Université de Montpellier II (France), 469p.

Richey JE., Melack JM., Aufdenkampe AK., Ballester VM., Hess LL. 2002. Outgassing from Amazonian rivers and wetlands as a large tropical source of atmospheric CO2. Nature 416:617-20.

Royer T.V., David M.B. 2005. Export of dissolved organic carbon from agricultural streams in Illinois, USA. Aquatic Sciences 67, 465-471.

Sanderman J., Lohse K.A., Baldock J.A., Amundson R. 2009. Linking soils and streams: sources and chemistry of dissolved organic matter in a small coastal watershed. Water Resources Research 45, W03418. 
doi:10.1029/2008WR006977.

Spencer R. G. M., Hernes P. J., Ruf R., Baker A., Dyda R. Y., Stubbins A. and Six J., 2010. Temporal controls on dissolved organic matter and lignin biogeochemistry in a pristine tropical river, Democratic Republic of Congo. Journal of Geophysical Research: Biogeosciences, 115, G03013. doi:10.1029/2009JG001180.

Spitzy A., and Leenheer, J.A., 1991. Dissolved organic carbon in rivers, in Degens, E.T., Kempe, Stephan, and Richey, J.E., eds., Biogeochemistry of major world rivers: New York, Wiley, p. 213-232.

St-Jean G. 2003. Automated quantitative and isotopic (13C) analysis of dissolved inorganic carbon and dissolved organic carbon in continuous-flow using a total organic carbon analyser, Rapid Communications in Mass Spectrometry, 17, 419-428.

Tamooh F., Van den Meersche K., Meysman F., Marwick T. R., Borges A. V., Merckx R., Dehairs F., Schmidt S., Nyunja J., et Bouillon S. 2012. Distribution and origin of suspended matter and organic carbon pools in the Tana River Basin, Kenya. Biogeosciences, 9: 2905-2920.

Tardy Y., Bustillo V., Boeglin J-L., 2004. Geochemistry applied to the watershed survey: hydrograph separation, erosion and soil dynamics. A case study: the basin of the Niger River, Africa. Applied Geochemistry 19: 469 518.

Thurman E. M., 1985. Organic carbon in natural waters: amount, origin and clasification, in: Organic geochemistry of natural waters, Kluwer Academic Publishers Dordrecht, Netherlands, 5-65.

Townsend S. A., Webster I. T. and Schult J. H., 2011. Metabolism in a groundwater-fed river system in the Australian wet/dry tropics: tight coupling of photosynthesis and respiration, Journal of the North American Benthologycol Sociaty, 30, 603-620.

Townsend-Small A., Noguera J. L., McClain M. E. and Brandes J. A., 2007. Radiocarbon and stable isotope geochemistry of organic matter in the Amazon headwaters, Peruvian Andes. Global Biogeochemical Cycles, Vol. 21, GB2029, doi:10.1029/2006GB002835

Veyssy E., 1998. Transferts de matières organiques des bassins versants aux estuaires de la Gironde et de l'Adour. Thèse Doctorat no 1789, Bordeaux 1, $262 \mathrm{pp}$.

Wasson J. G., Guyot J. L. et Sanejouand H., 1991. Premières données concernant le Carbone Organique transporté par le Rio Desaguadero (Altiplano Bolivien). Revue des sciences de l'eau / Journal of Water Science, vol. 4, n 3, p. 363-379. 